Fabio CiAnFeroni, Fabio TerZANi

\title{
Nuovi dati su Gerromorpha e Nepomorpha in Italia (Hemiptera Heteroptera)
}

\begin{abstract}
Riassunto: Vengono riportati nuovi dati geonemici italiani per dieci specie di Eterotteri acquatici. Gerromorpha, Veliidae: Velia currens (Fabricius, 1794), V. gridellii (Tamanini, 1947); Gerridae: Gerris asper (Fieber, 1860); Nepomorpha, Ochteridae: Ochterus marginatus (Latreille, 1804); Corixidae: Arctocorisa carinata (C.R. Sahlberg, 1819), Corixa panzeri Fieber, 1848, Hesperocorixa sahlbergi (Fieber, 1848), Parasigara perdubia (Rey, 1894), Sigara italica Jaczewski, 1933; Notonectidae: Anisops sardeus Herrich-Schäffer, 1849. Viene segnalata la prima popolazione appenninica di Arctocorisa carinata (C.R. Sahlberg, 1819).
\end{abstract}

\begin{abstract}
New records of Gerromorpha and Nepomorpha from Italy (Hemiptera Heteroptera).
New Italian geonemic records for ten species of aquatic Heteroptera are given. Gerromorpha, Veliidae: Velia currens (Fabricius, 1794), $V$. gridellii (Tamanini, 1947); Gerridae: Gerris asper (Fieber, 1860); Nepomorpha, Ochteridae: Ochterus marginatus (Latreille, 1804); Corixidae: Arctocorisa carinata (C.R. Sahlberg, 1819), Corixa panzeri Fieber, 1848, Hesperocorixa sahlbergi (Fieber, 1848), Parasigara perdubia (Rey, 1894), Sigara italica Jaczewski, 1933; Notonectidae: Anisops sardeus Herrich-Schäffer, 1849. The first apenninic population of Arctocorisa carinata (C.R. Sahlberg, 1819) is recorded.
\end{abstract}

Key words: Hemiptera, Heteroptera, Gerromorpha, Nepomorpha, Arctocorisa carinata, distribuzione.

\section{INTRODUZIONE}

Le conoscenze faunistiche sugli Emitteri Eterotteri acquatici e semi-acquatici (Nepomorpha e Gerromorpha) in Italia possono considerarsi relativamente buone, ma carenze sostanziali si riscontrano soprattutto per l'Italia centrale e meridionale (Cianferoni, 2011). Importanti contributi si devono, soprattutto, a Cesare Mancini (es. Mancini, 1952, 1963) che ha fornito numerosi apporti su varie faune regionali relative agli Eterotteri, riassunti successivamente da Servadei (1967), a Livio Tamanini con ricerche mirate su importanti aree geografiche (es. Tamanini, 1981, 1982) e attraverso la recente checklist di Bacchi \& Rizzotti Vlach $(2005,2007)$. Nonostante ciò, esistono ancora numerose lacune sulla distribuzione di queste specie in Italia e, inoltre, anche una pur grossolana distribuzione a carattere regionale risulta spesso difficile da ricavare a causa della presenza di numerosi lavori faunistici minori spesso ignorati nei contributi più recenti e, talvolta, della totale assenza di importanti faune riassuntive a carattere regionale (es. Toscana) o di un catalogo nazionale ragionato e aggiornato.

Dallo studio dei Gerromorfi e Nepomorfi provenienti soprattutto dalla collezione Terzani (recentemente donata al Museo di Storia Naturale dell'Università degli Studi di Firenze) sono emersi dati inediti e interessanti, che aggiungono un ulteriore tassello alla conoscenza di questi gruppi di insetti acquatici.

\section{Materiali e Metodi}

L'ordine sistematico seguito è quello di Schuh \& Slater (1995), mentre la nomenclatura fa riferimento ad Andersen (1995), Jansson (1995) e Polhemus (1995a, 1995b).

I corotipi, assegnati secondo Vigna Taglianti et al. (1993, 1999), derivano dalle geonemie presenti nel "Catalogue of the Heteroptera of the Palaearctic Region" (Aukema \& Rieger, 1995).

Le segnalazioni sono ordinate come segue: regione, corpo idrico, altitudine (metri s.l.m.), eventuale altra indicazione toponomastica, comune e provincia di appartenenza (sigla in parentesi), data, raccoglitore/i, numero di esemplari, eventuale indicazione dello stadio larvale, sesso ed eventuale indicazione sulla morfologia alare, collezione. L'elencazione delle regioni nella distribuzione italiana procede sostanzialmente da nord a sud e da ovest ad est, seguita poi dalla Sardegna (ad eccezione di Ochterus marginatus e Anisops sardeus a distribuzione prevalentemente meridionale, in cui l'elencazione inizia con la Sardegna e prosegue poi da sud a nord e da ovest ad est).

Le abbreviazioni utilizzate nel testo sono le seguenti:

apt=attero/i; macr $=$ macrottero/i; micr $=$ microttero/i; I$\mathrm{V}=$ larva di I-V stadio;

$\mathrm{CAM}=$ Collezione A. Mascagni, Scandicci (FI); 
$\mathrm{CFC}=$ Collezione $\mathrm{F}$. Cianferoni, Firenze;

$\mathrm{CFT}=$ Collezione F. Terzani (c/o MZUF);

MZUF=Museo di Storia Naturale dell'Università degli Studi di Firenze, Sezione di Zoologia "La Specola".

ELENCO FAUNISTICO

\section{GERROMORPHA}

\section{Veliidae}

Velia (Plesiovelia) currens (Fabricius, 1794)

Materiale esaminato. Umbria: Torrente Carpina, 410 m, Carpini, Montone (PG), 14.VIII.1990, S. Roc-

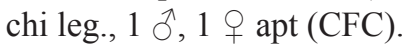

DistribuZione. Endemismo Alpino-Appenninico, presente in Italia continentale, Francia (Alpi Marittime), Svizzera (Canton Ticino), Austria meridionale e Slovenia (Cianferoni \& Santini, 2012). In base alle affinità filogenetiche (Vigna Taglianti et al., 1993) questo endemismo è riferibile ad un corotipo Europeo (Cianferoni \& Mazza, 2012). Già nota in tutte le regioni italiane continentali, con l'esclusione della Valle d'Aosta e dell'Umbria, è assente in Sicilia e Sardegna (Angelini, 1973; Fiordigigli \& Osella, 1994; Bacchi \& Rizzotti Vlach, 2005).

Note. Prima segnalazione per l'Umbria.

Velia (Plesiovelia) gridellii Tamanini, 1947

Materiale esaminato. Molise: Torrente Quirino, 800 m, Guardiaregia (CB), 2.VII.2003, F. Terzani leg., 3

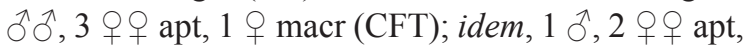
1 macr (CFC); Torrente Gamberale, $840 \mathrm{~m}$, Agnone, Isernia (IS), 15.VII.2011, F. Terzani \& F. Ceccolini leg., $3 \hat{\jmath}$ ऽ̂, 7 우 0 apt (CFT).

DistribUZIONE. Specie Alpino-Appenninica con areale alpino limitato all'arco delle Prealpi italiane fino alle Alpi Marittime franco-italiane. È presente all'Isola d'Elba (Tamanini, 1981; Bacchi \& Rizzotti Vlach, 2005). Questo endemismo è riferibile ad un corotipo Turanico-Mediterraneo, appartenendo ad un gruppo di specie che sembra trovare con questo taxon il limite occidentale del proprio areale distributivo (cfr. Cianferoni \& Mazza, 2012). Nota finora di tutte le regioni italiane escluse la Valle d'Aosta e il Molise; assente in Sicilia e Sardegna (Bacchi \& Rizzotti Vlach, 2005).

Note. Prima segnalazione per il Molise.

\section{Gerridae}

\section{Gerris (Gerriselloides) asper (Fieber, 1860)}

Materiale esaminato. Toscana: stagni retrodunali, Torre del Lago Puccini, Viareggio (LU), Parco Regionale di Migliarino San Rossore Massaciuccoli, 6.IV.1996, A. Mascagni leg., 1 o micr (CAM); stagni retrodunali, tra Marina di Torre del Lago Puccini e Viareggio (LU), Parco Regionale di Migliarino San Rossore Massaciuccoli, 2.IV.2007, F. Cianferoni leg.,

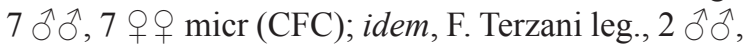
5 우 micr (MZUF), 1 O, 1 \& (CFT), 1 ㅇ (CFC); Padule di Fucecchio, Fucecchio (FI), Riserva Naturale Padule di Fucecchio, IV-V.1994, A. Bordoni leg., 1 ㅇ micr (CFC).

DisTRIBUZIONE. Specie a corotipo Europeo-Mediterraneo. In Italia è segnalata nelle regioni alpine orientali (Trentino-Alto Adige, Veneto e Friuli-Venezia Giulia) e con stazioni isolate in Toscana (Marina di Torre del Lago Puccini; Isola d'Elba), Lazio (Roma, Piazza d'Armi; Lago di Fondi) e Basilicata (Policoro) (Marcuzzi \& Lorenzoni, 1970; Bacchi \& Rizzotti Vlach, 2005; Dionisi, 2007; Cianferoni et al., 2013).

Note. I presenti dati confermano la presenza della specie in Versilia (Toscana), dove era già stata raccolta nel 1957 (Bacchi \& Rizzotti Vlach, 2005), e aggiungono una nuova stazione toscana (Padule di Fucecchio). La specie è dimorfa con forma microttera e macrottera. Il morfotipo non volatore è microttero e non attero come indicato da Tamanini (1979), infatti le ali, estremamente ridotte, risultano ancora visibili ai lati della base del pronoto.

\section{NEPOMORPHA}

\section{Ochteridae}

Ochterus (Ochterus) marginatus marginatus (Latreille, 1804)

Materiale eSAminato. Toscana: Torrente Sterza, 80140 m, Laiatico e Chianni (PI), 10.IX.2007, F. Terzani leg., 1 V, 1 IV (CFT).

DistRIBUZIONE. Si tratta di una specie ad ampia distribuzione che abbraccia la regione Paleartica, Afrotropicale e Orientale; nel Paleartico occidentale è presente nell'area mediterranea (Kormilev, 1973; Polhemus, 1995a; Chen et al., 2005). In Italia si ritrova con maggiore frequenza nelle regioni meridionali, mentre appare molto più sporadica procedendo verso quelle settentrionali. La reale distribuzione italiana ri- 
sulta ancora incompleta; ad oggi risulta nota per Sicilia, Calabria, Basilicata, Campania, Abruzzo, Lazio, Umbria, Marche, Liguria, Piemonte e Trentino-Alto Adige; non è presente in Sardegna, né su alcuna delle isole minori (Servadei, 1967; Bacchi \& Rizzotti Vlach, 2005).

Note. Prima segnalazione per la Toscana. Questo dato è importante per completare la distribuzione italiana di questa specie, fungendo da collegamento tra una segnalazione del 1990 per il Lazio (comune di Roma) riportata in Dionisi (2007) e quella di Mancini (1952) per la Liguria (Albenga). Ochterus marginatus (Latreille, 1804) sembrerebbe costituire un complex di specie (N. Nieser, in litteris), comprendente alcune specie criptiche occupanti ciascuna una porzione dell'ampio areale (Chen et al., 2005). Almeno in parte tale situazione è stata chiarita da Polhemus \& Polhemus (2012) attraverso la descrizione di alcune nuove specie. Gli esemplari delle popolazioni più orientali (Filippine e Bali), caratterizzate da minori dimensioni e da differenze nel paramero destro e nel processo medio del pigoforo, vengono attualmente assegnate alla sottospecie O. m. insularis Rieger, 1977, in attesa di ulteriori studi (Gapud, 2003; Polhemus \& Polhemus, 2012).

\section{Corixidae}

Arctocorisa carinata carinata (C.R. Sahlberg, 1819)

Materiale esaminato. Toscana: Lago Nero, $1730 \mathrm{~m}$,

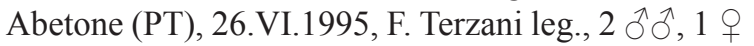
macr (CFT); idem, 1 ô macr (CFC).

DistRIBUZIONE. Specie boreo-alpina inquadrabile in un corotipo Sibirico-Europeo (Vigna Taglianti et al. 1993, 1999). Esistono popolazioni in Islanda, nelle Isole Far Oer, in Scozia e Inghilterra settentrionale, sulle coste del Baltico e dalle coste del Mar del Nord, lungo la Scandinavia e attraverso Lapponia e Urali fino al bacino del Fiume Ob. Nel territorio dell'Altaj esiste una sottospecie distinta morfologicamente: $A$. carinata lansburyi Jansson, 1979. Vi sono poi tre popolazioni isolate nelle regioni montane meridionali inquadrabili come relitti glaciali: sui Pirenei, sulle Alpi e sui Monti Balcani (Jansson, 1986). In Italia la specie è citata lungo tutto l'arco alpino (Liguria, Piemonte, Valle d'Aosta, Lombardia, Trentino-Alto-Adige e Veneto) (Mancini, 1963; Bacchi \& Rizzotti Vlach, 2005).

Note. Gli esemplari sono stati raccolti nel Lago Nero, un lago di origine glaciale dell'Appennino Toscano.
Il ritrovamento è da considerarsi di estremo interesse dato che si tratta della prima popolazione appenninica finora nota. Le stazioni alpine più vicine sono quelle del bacino del F. Roia, nel Nizzardo (Mancini, 1963) e quelle in Val Corsaglia, Piemonte (Bacchi \& Rizzotti Vlach, 2005). La popolazione alpina (testata in base ad esemplari della Svizzera) è risultata essere riproduttivamente isolata e separata dal punto di vista morfologico (in base ai caratteri sessuali maschili) ed è da considerarsi attualmente in corso di speciazione (Jansson, 1978; Jansson \& Pajunen, 1978). Ulteriori indagini sarebbero necessarie per stabilire l'affinità genetica con le popolazioni alpine ed un eventuale isolamento riproduttivo. La reale dimensione di questa popolazione è attualmente sconosciuta, tuttavia, in base all'estensione del bacino lacustre $\left(0,08 \mathrm{~km}^{2}\right) \mathrm{e}$ alla apparente assenza nei biotopi circostanti, tutte le precauzioni del caso andrebbero osservate nell'effettuare eventuali studi (es. prelievo di un numero minimo di esemplari e allevamento in laboratorio). I presenti dati costituiscono la prima segnalazione per la Toscana.

\section{Corixa panzeri Fieber, 1848}

Materiale esaminato. Toscana: Oasi della Cornacchiaia, Tirrenia, Pisa (PI), Parco Regionale di Migliarino San Rossore Massaciuccoli, 27.VI.2008, L. Giugliano leg., 1 ô, 1 + macr (CFC); Spiaggia della Lecciona, Torre del Lago Puccini, Viareggio (LU), Parco Regionale di Migliarino San Rossore Massaciuccoli, 28.VI.2008, L. Giugliano leg., 1 q macr (CFC). Sardegna: pozze dietro la diga del Lago Posada, Torpè (NU), 23.VII.1978, R. Brizzi, G. Guarducci \& R. Innocenti leg., $4 \widehat{\delta} \widehat{\partial}, 10$ 우 0 macr (MZUF).

DistRIBUZIONE. Specie Turanico-Europeo-Mediterranea con estensione all'Europa centro-settentrionale. In Italia le segnalazioni per questa specie sono scarse; è attualmente nota per Piemonte, Trentino-Alto Adige, Veneto, Liguria, Emilia-Romagna, Toscana, Umbria, Basilicata, Puglia, Calabria, Sicilia e Sardegna (Bacchi \& Rizzotti Vlach, 2005; Ansaloni et al., 2010). Presente anche in Corsica (Jansson, 1986).

Note. Questi dati si aggiungono alle limitate e sporadiche segnalazioni esistenti per questo taxon. I presenti dati per la Toscana costiera settentrionale si posizionano tra le segnalazioni liguri e quelle della Toscana costiera meridionale (Bacchi \& Rizzotti Vlach, 2005). 
Hesperocorixa sahlbergi (Fieber, 1848)

Materiale esaminato. Toscana: Paduletta di Ramone, Padule di Fucecchio, Larciano (PT), Riserva Naturale Padule di Fucecchio, 5.IX.1993, F. Terzani leg., 1 乃 , 2 우우 macr (CFT); Padule di Fucecchio, Fucecchio (FI), Riserva Naturale Padule di Fucecchio, IV-V.1994, A. Bordoni leg., 2 đิ ô macr (CFC); Lago di Porta, Montignoso (MS), ANPIL "Lago di Porta", 27.V.2008, L. Giugliano leg., 1 Oे (CFC). Molise: Palude della Zittola, Montenero Valcocchiara (IS), 13.VIII.2008, R. Merciai, S. Bertocchi \& S. Brusconi leg., 1 ô, 1 V (CFC).

Distribuzione. Specie Sibirico-Europea, nota di tutte le regioni dell'Italia continentale con l'esclusione di Valle d'Aosta e Marche (Servadei, 1967; Fiordigigli \& Osella, 1994; Bacchi \& Rizzotti Vlach, 2005). Non è presente sulle isole italiane (Tamanini, 1979), ma Jansson (1986) la indica di Corsica.

NoTE. A causa delle scarsità e sporadicità delle segnalazioni italiane, vengono forniti nuovi dati per la Toscana, dove la specie era finora nota soltanto dell'area di Viareggio e ne viene riconfermata la presenza nella Palude della Zittola (Bacchi \& Rizzotti Vlach, 2005).

\section{Parasigara perdubia (Rey, 1894)}

Materiale esaminato. Toscana: Botro Campo di Sasso, 68 m, Bibbona (LI), ANPIL "Macchia della

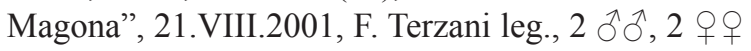
macr (CFT); idem, 1 ô, 1 q macr (CFC); Botro Campo di Sasso, 110 m, Bolgheri, Castagneto Carducci (LI), ANPIL "Macchia della Magona", 17.X.2001, F. Terzani leg., 1 ô, 2 q $ᄋ$ macr (CFT); Isola di Capraia, Capraia Isola (LI), 1-2.V.1982, S. Taiti \& S. Vanni leg., 3 우 macr (MZUF, det. A. Jansson); Poggio Cavallo, Grosseto (GR), XII.1899, A. Andreini leg. [Coll. Andreini / Poggio Cavallo / dint. Grosseto / XII.1899], 2 ঐึ A. Jansson).

DistribuZIONE. Elemento W-Mediterraneo, assente in Maghreb. In Italia è segnalato per Liguria, Toscana continentale e insulare (isole Elba e Capraia), Lazio e Sardegna (Cianferoni, 2011). Presente anche in Corsica (Jansson, 1986).

Note. Si riportano i primi dati con riferimento toponomastico preciso per la Toscana continentale, dove la presenza di questa specie nella regione era deducibile soltanto dalla mappa pubblicata da Jansson (1986: 53).
Jansson (l.c.) indica anche un sito dell'Arcipelago Toscano, probabilmente coincidente con gli esemplari dell'Isola di Capraia determinati dallo stesso autore e presenti nella Collezione del Museo di Storia Naturale dell'Università di Firenze (MZUF). Una conferma per l'Isola di Capraia risultava comunque già da una ulteriore segnalazione di Bacchi \& Rizzotti Vlach (2005).

\section{Sigara (Subsigara) italica Jaczewski, 1933}

Materiale esaminato. Emilia-Romagna: Lago Moo, 1100 m, Ferriere (PC), 16.VII.2009, F. Terzani \& S. Rocchi leg., 1 đิ macr (CFT); Lago di Pratignano, 1307 m, Fanano (MO), 4.VI.2009, F. Terzani \& S. Rocchi leg., 4 §ô, 1 ㅇ macr (CFT); idem, S. Rocchi \& F. Terzani leg., 4 $\hat{\jmath}, 1$ q macr (CFC).

Distribuzione. Endemismo italiano, vicariante della specie europea Sigara (Subsigara) falleni (Fieber, 1848) (Jansson, 1986). Risulta presente in tutte le regioni dell'Italia settentrionale (esclusa la Valle d'Aosta), Toscana e Lazio (Melber, 1993; Bacchi \& Rizzotti Vlach, 2005; Dionisi, 2007).

Note. Le presenti segnalazioni confermano la presenza di S. italica in Emilia-Romagna (Appennino emiliano), dove era già citata da Servadei (1967) per la pianura bolognese.

Sigara (Subsigara) falleni (Fieber, 1848) è citata per l'Italia settentrionale (Servadei, 1967; Tamanini, 1979; cfr. Jansson, 1986), ma tali segnalazioni non vengono riportate da Bacchi \& Rizzotti Vlach (2005). Questi autori indicano un'unica segnalazione italiana (Toscana: Pontassieve / $100 \mathrm{~m} /$ Coll. Mus. Civ. St. Nat. Verona / 1987), citata anche da Dionisi (2007). Questo materiale non è però presente al Museo Civico di Storia Naturale di Verona (R. Salmaso, in litteris). La località è compatibile con le ricerche di Melber (1993) in Toscana, il quale ha depositato parte degli esemplari nel suddetto museo. Tale autore non riporta però Sigara falleni tra i risultati delle sue ricerche, ma soltanto Sigara (Subsigara) italica e Sigara (Subsigara) fossarum (Leach, 1817) (costituenti le prime segnalazioni per la Toscana). Entrambi i taxa sono stati verificati dal primo autore (FC) in base al materiale conservato nella propria collezione (Cianferoni \& Mazza, 2012) e in quella del Museo Civico di Storia Naturale di Verona.

In considerazione di quanto sopra riteniamo, per il momento, di poter escludere Sigara falleni dalla fauna toscana. La presenza in Italia risulta comunque ancora 
supportata almeno dal dato per l'Italia occidentale di Jansson (1986: 80) deducibile da mappa. Inoltre la presenza di $S$. falleni sulle sponde del Lago Maggiore (Canton Ticino, Svizzera) riportata da Giacalone et al. (2002) sembrerebbe confermare l'esistenza della specie almeno in Lombardia (Servadei, 1967). Mentre i dati (precedenti la descrizione di S. italica) per il Friuli-Venezia Giulia, riportati da Servadei (1967) e Dioli (1987), necessiterebbero di controllo o riconferma.

\section{Notonectidae}

Anisops sardeus sardeus Herrich-Schäffer, 1849

Materiale esaminato. Toscana: stagni retrodunali, Spiaggia della Lecciona, $2 \mathrm{~m}$, Torre del Lago Puccini, Viareggio (LU), Parco Regionale di Migliarino San Rossore Massaciuccoli, 7.VIII.2008, L. Giugliano leg., 1 (CFC); laghetto, loc. "i Piani”, 645 m, presso Rincine, Londa (FI), 30.VII.2009, F. Cianferoni leg., 1 ô, 3 V (CFC); Lago Novo, $41 \mathrm{~m}$, Galciana (PO), 22.IV.2010, S. Rocchi, F. Cianferoni \& F. Terzani leg., 1 ㅇ (CFC).

DistribuZIONE. Specie ad ampia distribuzione, presente dalla regione Afrotropicale fino a quella mediterranea, ad oriente si spinge fino a India e Myanmar. Risulta più comune nelle regioni dell'Italia meridionale e insulare, mentre diviene progressivamente più sporadica procedendo verso nord. È attualmente nota per le seguenti regioni italiane: Sardegna, Sicilia, Calabria, Puglia, Basilicata, Campania, Lazio, Toscana, Emilia-Romagna e Veneto. È inoltre presente in numerose isole minori, in Corsica e nelle Isole Maltesi (Cianferoni \& Pinna, 2012).
Note. Questi ritrovamenti risultano di notevole interesse per comprendere meglio la distribuzione italiana e toscana della specie. Il dato per la provincia di Lucca sposta più a nord l'areale costiero della specie, che fino a questo momento era nota, per la Toscana, solo di due stazioni del "Parco Regionale della Maremma", provincia di Grosseto (Bacchi \& Rizzotti Vlach, 2005). Gli esemplari dell'Appennino toscano e della Piana fiorentina dimostrano come tale specie possa spingersi in zone interne anche nell'Italia centro-settentrionale. Considerando anche i recenti ritrovamenti per il Veneto (Cianferoni \& Pinna, 2012), l’Ungheria (Soós et al., 2010), la Romania (Berchi, 2011) e la Repubblica russa di Cabardino-Balcaria (Khatukhov et al., 2008), si può concordare con l'ipotesi di una progressiva espansione verso nord di questa specie (Berchi, 2008) e inquadrarla in un più generale effetto di "mediterraneizzazione" della fauna europea (Rabitsch, 2008).

\section{RINGRAZIAMENTI}

Desideriamo ringraziare Luca Bartolozzi (MZUF) per averci permesso di studiare la collezione museale; Arnaldo Bordoni, Filippo Ceccolini, Alessandro Mascagni e Saverio Rocchi (MZUF), Luigi Giugliano, Silvia Bertocchi, Sara Brusconi (Dipartimento di Biologia Evoluzionistica "Leo Pardi", Università degli Studi di Firenze), Roberto Merciai (Institut d'Ecologia Aquàtica, Universitat de Girona, Spagna) per averci permesso di studiare le proprie collezioni e/o donato alcuni esemplari; Filippo Fabiano, Giuseppe Mazza e Francesca Zinetti (MZUF) per l'aiuto nelle raccolte di materiale durante le ricerche. 


\section{BIBLIOGRAFIA}

Andersen N.M., 1995 - Infraorder Gerromorpha Popov, 1971 - semiaquatic bugs (pp. 77-114). In: AukEMA B. \& RIEGER C. (eds). Catalogue of the Heteroptera of the Palaearctic Region. Volume 1. Enicocephalomorpha, Dispocoromorpha, Nepomorpha, Gerromorpha and Leptopodomorpha. The Netherlands Entomological Society, Wageningen, 222 pp.

ANGELINI F., 1973 - Emitteri Eterotteri acquatici nuovi o interessanti per Puglia e Lucania. Bollettino della Società entomologica italiana, 105 (9-10): 143-148.

AnSAloni I., ARTioli P. \& SimOnini R., 2010 - La comunità macrozoobentonica di alcune zone umide del Modenese: III - "Valli le Partite" (Comune di Mirandola). Atti della Società dei naturalisti e matematici di Modena, 140 (2009): 153-165.

Aukema B. \& Rieger C., 1995 - Catalogue of the Heteroptera of the Palaearctic Region. Volume 1. Enicocephalomorpha, Dispocoromorpha, Nepomorpha, Gerromorpha and Leptopodomorpha. The Netherlands Entomological Society, Wageningen, $222 \mathrm{pp}$.

Bacchi I. \& Rizzotti Vlach M., 2005 - Insecta Heteroptera Nepomorpha e Gerromorpha (pp. 147-149). In: Ruffo S. \& Stoch F. (eds). Checklist e distribuzione della fauna italiana. Memorie del Museo civico di Storia naturale di Verona, 2. Serie, Sezione Scienze della Vita, 16, 307 pp. + CD-ROM.

Bacchi I. \& Rizzotti Vlach M., 2007 - Insecta Heteroptera Nepomorpha and Gerromorpha (pp. 147-149). In: RUFFo S. \& Sтосн F. (eds). Checklist and Distribution of the Italian Fauna. Memorie del Museo civico di Storia naturale di Verona, 2. Serie, Sezione Scienze della Vita, 17 (2006), 303 pp. + CD-ROM.

BerCHI G.M., 2011 - First record of Anisops sardeus (Hemiptera: Heteroptera: Notonectidae) in Romania. North-Western Journal of Zoology, 7 (2): 339-341.

Chen P.-P., Nieser N. \& Zettel H., 2005 - The aquatic and semi-aquatic bugs (Heteroptera, Nepomorpha \& Gerromorpha) of Malesia. Fauna Malesiana Handbook, 5. Brill Academic Publisher, 546 pp.

Cianferoni F., 2011 - Notes on Gerromorpha, Nepomorpha and Leptopodomorpha from Sardinia (Hemiptera, Heteroptera) (pp. 255-268). In: Nardi G., Whitmore D., Bardiani M., Birtele D., Mason F., Spada L. \& Cerretti P. (eds). Biodiversity of Marganai and Montimannu (Sardinia). Research in the framework of the ICP Forests network. Conservazione Habitat Invertebrati, 5. Cierre Edizioni, Sommacampagna, Verona, $896 \mathrm{pp}$.

Cianferoni F. \& Mazza G., 2012 - The aquatic Heteroptera (Insecta: Hemiptera) of the "Foreste Casentinesi, Monte Falterona e Campigna" National Park (Central Italy). Zootaxa, 3568: 36-52.

Cianferoni F. \& Pinna A., 2012 - Segnalazioni faunistiche italiane. 529 - Anisops sardeus sardeus Herrich-Schäffer, 1849 (Hemiptera Heteroptera Notonectidae). Bollettino della Società entomologica italiana, 144 (1): 44.

Cianferoni F. \& SAntini G., 2012 - Ecology and life histories of two Alpine-Apenninic species of Velia Latreille (Hemiptera: Heteroptera: Veliidae). European Journal of Entomology, 109: 427-434.

Cianferoni F., Rocchi S. \& Terzani F., 2013 - Nepomorpha, Gerromorpha and Leptopodomorpha (Hemiptera: Heteroptera) of the Tuscan Archipelago (Italy). Zootaxa, 3669 (3): 302-320.

Dioli P., 1987 - Il popolamento degli Eterotteri (Insecta Heteroptera) in Friuli e Venezia Giulia. Biogeographia, 13: 605-619.

Dionisi R., 2007 - Gli Eterotteri acquatici del Lazio (Heteroptera: Gerromorpha, Nepomorpha). Bollettino dell'Associazione romana di Entomologia, 62 (1-4): 41-100.

Fiordigigli R. \& Osella G., 1994 - Ricerche faunistiche sulla Palude della Zittola (Abruzzo-Molise). II. Il popolamento ad Emitteri Eterotteri. Quaderni della Stazione di Ecologia del civico Museo di Storia naturale di Ferrara, 6: 27-80.

GAPUD V.P., 2003 - Two new Philippine Ochterus Latreille (Insecta: Heteroptera: Ochteridae) and checklist of Philippine species. Annalen des Naturhistorischen Museums in Wien, Serie B, 104 (2002): 99-108.

Giacalone I., Dioli P. \& PAtocchi N., 2002 - Monitoraggi faunistici alle Bolle di Magadino (Svizzera meridionale) eterotteri acquatici terrestri (Insecta, Heteroptera: Gerromorpha, Nepomorpha e Geocoridae). Bollettino della Società ticinese di Scienze naturali, 90 (1-2): 81-92.

Jansson A., 1986 - The Corixidae (Heteroptera) of Europe and some adjacent regions. Acta Entomologica Fennica, 47: 1-94.

JANSSON A., 1995a - Family Corixidae Leach, 1815 - water boatmen (pp. 26-56). In: AukemA B. \& RiEgER C. (eds). Catalogue of the Heteroptera of the Palaearctic Region. Volume 1. Enicocephalomorpha, Dispocoromorpha, Nepomorpha, Gerromorpha and Leptopodomorpha. The Netherlands Entomological Society, Wageningen, 222 pp.

JANSSON A. \& PAJUNEN V.I., 1978 - Morphometric comparison of geographically isolated populations of Arctocorisa carinata (C. Sahlberg) (Heteroptera, Corixidae). Annales Zoologici Fennici, 15: 132-142. 
Khatukhov A.M., Yakimov A.V. \& Lvov V.D., 2008 - Backswimmers (Heteroptera: Notonectidae) of the Central Caucasus (territory of the Kabardino-Balkarian Republic). Bulletin of the Adyghe State University, Maikop, 4: 117-119.

KormiLev N.A., 1973 - Ochteridae from Western and Southern Africa (Hemiptera: Heteroptera). Occasional papers of the California Academy of Sciences, 106: 1-9.

MANCINI C., 1952 - Miscellanea emitterologica italiana. I. Bollettino della Società entomologica italiana, 82: 56-64.

MAnCINI C., 1963 - Emitteri Eterotteri della Liguria. Res Ligusticae CXXXIII. Annali del Museo Civico di Storia Naturale "G. Doria”, 74: 30-121.

MarcuzZi G. \& Lorenzoni A.M., 1970 - Osservazioni ecologico-faunistiche sul popolamento animale della Palude carsica di Pietra Rossa (Monfalcone) (II nota). Vie et Milieu, 21 (1-C): 1-58.

Melber A., 1993 - Beitrag zur Kenntnis der Heteropterenfauna des toskanischen Apennine (Insecta, Heteroptera). Bollettino del Museo Civico di Storia Naturale di Verona, 17 (1990): 293-356.

Polhemus J.T., 1995a - Family Ochteridae Kirkaldy, 1906 - velvety shore bugs (pp. 25-26). In: AuKema B. \& Rieger C. (eds). Catalogue of the Heteroptera of the Palaearctic Region. Volume 1. Enicocephalomorpha, Dispocoromorpha, Nepomorpha, Gerromorpha and Leptopodomorpha. The Netherlands Entomological Society, Wageningen, $222 \mathrm{pp}$.

Polhemus J.T., 1995b - Family Notonectidae Latreille, 1802 - backswimmers (pp. 63-73). In: AukEMA B. \& RIEGER C. (eds). Catalogue of the Heteroptera of the Palaearctic Region. Volume 1. Enicocephalomorpha, Dispocoromorpha, Nepomorpha, Gerromorpha and Leptopodomorpha. The Netherlands Entomological Society, Wageningen, 222 pp.

Polhemus D.A. \& Polhemus J.T., 2012 - Guide to the Aquatic Heteroptera of Singapore and Peninsular Malaysia. IX. Infraorder Nepomorpha, families Ochteridae and Gelastocoridae. The Raffles Bulletin of Zoology, 60 (2): 343-359.

RABITSCH W., 2008 - The times they are a-changin': driving forces of recent additions to the Heteroptera fauna of Austria (pp. 309-326). In: Grozeva S. \& Simov N. (eds). Advances in Heteroptera Research. Festschrift in Honour of 80th Anniversary of Michail Josifov. Pensoft Publishers, Sofia-Moscow, 417 pp.

Schun R.T. \& Slater J.A., 1995 - True Bugs of the World (Hemiptera: Heteroptera). Classification and Natural History. Cornell University Press, Ithaca, New York, I-XII + 336 pp.

Servadei A., 1967 - Rhynchota: Heteroptera. Homoptera. Auchenorrhyncha. Fauna d'Italia. 9. Calderini, Bologna, 854 pp.

Soós N., Petri A., NAgY-LÁszló Z. \& Csabai Z., 2010 - Anisops sardeus Herrich-Schaeffer, 1849: first records from Hungary (Heteroptera: Notonectidae). Folia Entomologica Hungarica, 71: 15-18.

TAMANINI L., 1979 - Eterotteri acquatici (Heteroptera: Gerromorpha, Nepomorpha). Guide per il riconoscimento delle specie animali delle acque interne italiane. CNR, AQ/1/43, 6: 1-106.

TAMAnini L., 1981 - Gli Eterotteri della Basilicata e della Calabria. Italia meridionale (Hemiptera Heteroptera). Memorie del Museo Civico di Storia Naturale di Verona. 2. Serie. Sezione Scienze della Vita (A: Biologica), 3: 1-164.

TAmanini L., 1982 - Gli Eterotteri dell'Alto Adige (Insecta: Heteroptera). Studi Trentini di Scienze Naturali. Acta Biologica, 59: 65-194.

Vigna Taglianti A., Audisio P.A., Belfiore C., Biondi M., Bologna M.A., Carpaneto G.M., De Biase A., De Felici S., Piattella E., Racheli T., Zapparoli M. \& Zoia S., 1993 - Riflessioni di gruppo sui corotipi fondamentali della fauna W-paleartica ed in particolare italiana. Biogeographia, Lavori della Società italiana di Biogeografia (n. s.), 16 (1992): 159-179.

Vigna Taglianti A., Audisio P.A., Biondi M., Bologna M.A., Carpaneto G.M., De Biase A., Fattorini S., Piattella E., SindACo R., VENCHI A. \& ZAPPAROLI M., 1999 - A proposal for chorotype classification of the Near East fauna, in the framework of the Western Palearctic region. Biogeographia. Lavori della Società italiana di Biogeografia (n. s.), 20: 31-59.

Indirizzo degli autori:

Fabio Cianferoni, Fabio Terzani, Museo di Storia Naturale dell’Università degli Studi di Firenze, Sezione di Zoologia "La Specola”, via Romana 17, 50125, Firenze, Italia. E-mail: fabio.cianferoni@unifi.it 